TUM-T31-79/94

hep-ph 9409454

September 1994

\title{
Two-loop mixing of dimension-five flavor-changing operators:
}

\author{
Mikołaj Misiak[ and Manfred Münz \\ Physik-Department, Technische Universität München \\ D-85747 Garching, Germany
}

\begin{abstract}
We calculate the two-loop QCD mixing of the dimension-five flavor-changing operators that arise in the Standard Model after integrating out the $W, Z$ and Higgs bosons and the topquark, i.e. the mixing among gluonic and photonic magnetic moment operators. Our calculation completes the two-loop anomalous dimension matrix of operators that govern low energy flavorchanging processes. It is an important ingredient of the next-to-leading calculation of the $B \rightarrow X_{s} \gamma$ decay rate.
\end{abstract}

*Supported by the German Bundesministerium für Forschung und Technologie under contract 06 TM 732, by the CEC Science project SC1-CT91-0729 and by the Polish Committee for Scientific Research.

${ }^{\dagger}$ On leave of absence from the Institute of Theoretical Physics, Warsaw University. 


\section{Introduction}

Flavor changing processes at energies much below the electroweak scale are most conveniently described with help of the effective hamiltonian [1]

$$
H_{e f f} \sim \sum_{i} C_{i}(\mu) O_{i}(\mu)
$$

where $O_{i}$ are dimension-five and -six operators built out of light $(u, d, s, c$ and $b)$ quarks, leptons, photons and gluons, and $C_{i}(\mu)$ are their Wilson coefficients. The operators $O_{i}$ that remain after applying the equations of motion (EOM) [2] are dimension-six four-fermion operators and the dimension-five magnetic moment operators:

$$
\begin{aligned}
O_{\gamma} & =e \bar{q} \sigma^{\mu \nu} F_{\mu \nu} q^{\prime} \\
O_{g} & =g \bar{q} \sigma^{\mu \nu} G_{\mu \nu}^{a} T^{a} q^{\prime}
\end{aligned}
$$

Here $F_{\mu \nu}$ and $G_{\mu \nu}^{a}$ denote the photonic and gluonic field strength tensors, respectively, and $T^{a}$ are the color group generators. The quarks $q$ and $q^{\prime}$ have different flavor and usually have specific chiralities but, as explained below, this is irrelevant in our calculation. The QED and QCD coupling constants are denoted by $e$ and $g$, respectively.

The factorization of short- and long-distance QCD effects is achieved [1] by evolving the coefficients $C_{i}(\mu)$ from the electroweak scale down to the low energy scale of the process under consideration. The anomalous dimension matrix that governs this evolution is known up to two loops for the QCD mixing of the phenomenologically important 4-quark operators into themselves [3], quark-lepton operators [4] and the magnetic moment operators [2, 5, 6]. The only remaining two-loop QCD mixing among all these operators is the two-loop mixing of the magnetic moment operators (目) and (3) which we calculate in the present paper.

Our results can have many phenomenological applications, as they affect all possible flavorchanging processes involving two quarks and a photon or a gluon. The two-loop mixing we calculate can also be directly applied to flavor-conserving magnetic moment operators (see end of section 3). However, the most important application of our results is their use in the calculation of the complete next-to-leading logarithmic QCD corrections to the $B \rightarrow X_{s} \gamma$ decay rate. The critical phenomenological importance of calculating the next-to-leading QCD corrections to the $B \rightarrow X_{s} \gamma$ rate has been stressed in the previous paper [7]. We will not 
repeat this discussion here. Instead, we will put more emphasis on the details of the present calculation.

The paper is organized as follows: In the next section, we recalculate the one-loop mixing of $O_{\gamma}$ and $O_{g}$. We recover also the nonphysical one-loop counterterms that will be needed for cancellation of subdivergences in the further two-loop computation. Section 3 presents certain details of the two-loop calculation. Our final results are given in the end of this section. In the last section, we rewrite our results in the notation common for the analyses of the $b \rightarrow s \gamma$ decay and show the size of the next-to-leading contributions we have calculated.

\section{The one-loop calculation}

Our starting point is the lagrangian density

$$
\mathcal{L}=\mathcal{L}_{Q C D \times Q E D}+X\left(C_{\gamma} O_{\gamma}+C_{g} O_{g}+\sum_{k} \tilde{C}_{k} \tilde{O}_{k}\right)
$$

Here $\mathcal{L}_{Q C D \times Q E D}$ is the full $Q C D \times Q E D$ lagrangian for $\mathrm{f}$ massless $\mathrm{Q}$ flavors, including the gauge fixing and ghost terms. X denotes some normalization constant that does not get QCDrenormalized. The operators $\tilde{O}_{k}$ are additional dimension-five operators required as counterterms for off-shell Green's functions with insertions of $O_{\gamma}$ and $O_{g}$. According to the standard textbook by Collins [9], these operators either vanish by EOM or are BRS-exact (i.e. are BRS variations of some other operators), and are therefore nonphysical. In the following, we will assume nothing about these operators but just recover them from the explicit one-loop calculation.

We have performed our calculation off-shell in the background field [8] version of the Feynman-'t Hooft gauge. We have calculated UV-divergent parts of one-particle-irreducible (1PI) one-loop diagrams with single $O_{\gamma}$ or $O_{g}$ insertions. All such diagrams with nonnegative degree of divergence correspond to the following transitions: $q^{\prime} \rightarrow q, q^{\prime} \rightarrow q \gamma, q^{\prime} \rightarrow q Q, q^{\prime} \rightarrow q A$, $q^{\prime} \rightarrow q \gamma \gamma, q^{\prime} \rightarrow q \gamma Q, q^{\prime} \rightarrow q \gamma A, q^{\prime} \rightarrow q Q Q, q^{\prime} \rightarrow q Q A$ and $q^{\prime} \rightarrow q A A$, where $\mathrm{Q}$ and A denote the quantum and the background gluon fields, respectively. The diagrams corresponding to the first three transitions are shown in fig. 1.

Since QCD and QED interactions preserve chirality, it is obvious that the mixing we calculate is independent of whether the operators $O_{\gamma}$ and $O_{g}$ are taken as they stand in eqs. (2) and

\footnotetext{
${ }^{1}$ Introducing fermion masses would not affect the mixing among $O_{\gamma}$ and $O_{g}$ calculated here.
} 


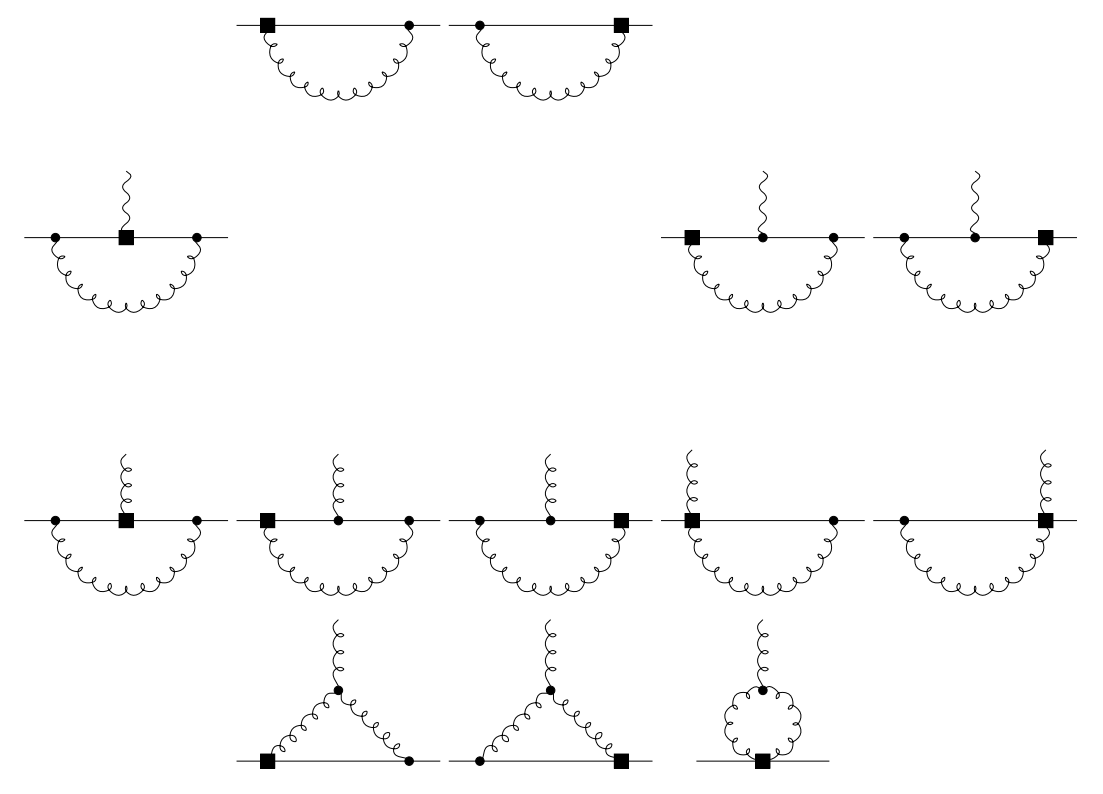

Figure 1: One-loop diagrams for the $q^{\prime} \rightarrow q, q^{\prime} \rightarrow q \gamma$ and $q^{\prime} \rightarrow q Q$ transitions. The squares denote insertions of $O_{\gamma}$ or $O_{g}$.

([) or specific (opposite) chiralities for the quarks $q$ and $q^{\prime}$ are chosen, as it happens in the phenomenologically important case of the $b \rightarrow s \gamma$ decay. In the following, we will take these operators as they stand in eqs. (2) and (3).

We find that divergences in all the possible one-loop 1PI Green's functions with single $O_{\gamma}$ or $O_{g}$ insertions can be removed by counterterms proportional to $O_{\gamma}$ and $O_{g}$ themselves, and to the following two operators:

$$
\begin{aligned}
& \tilde{O}_{D D}=\bar{q} \not D \not q^{\prime} \\
& \tilde{O}_{Q D}=i g \bar{q}(-\overleftarrow{D} \not \varnothing+\not \not D) q^{\prime}
\end{aligned}
$$

where $D_{\mu}$ is the usual $Q C D \times Q E D$ covariant derivative.

Assembling the four operators into the vector $\left(O_{\gamma}, O_{g}, \tilde{O}_{D D}, \tilde{O}_{Q D}\right)^{T}$, the corresponding oneloop anomalous dimension matrix reads

$$
\hat{\gamma}=\frac{g^{2}}{16 \pi^{2}}\left(\begin{array}{cc|cc}
2 C_{f} & 0 & 0 & 0 \\
8 C_{f} Q_{q} & 10 C_{f}-4 N & -12 C_{f} & \frac{3}{2} N \\
\hline 0 & 0 & ? & ? \\
0 & 0 & ? & ?
\end{array}\right)+\mathcal{O}\left(g^{4}\right)
$$

where $N$ denotes the number of colors, $C_{f}=\left(N^{2}-1\right) / 2 N$ and $Q_{q}$ is the electric charge of the $q$-quark (which must be identical to the one of $q^{\prime}$ ). The mixing of $O_{\gamma}$ and $O_{g}$ among 
themselves, as well as the mixing of $O_{g}$ into $\tilde{O}_{D D}$ are in agreement with what has been found previously [5, 10]. Note that in refs. [5, 10] and in most other papers, the quark mass is introduced in the normalization of $O_{\gamma}$ and $O_{g}$. So $\hat{\gamma}_{\text {here }}=\hat{\gamma}_{\text {there }}+\gamma_{m} \hat{1}$, where $\gamma_{m}=-\frac{g^{2}}{16 \pi^{2}} 6 C_{f}+\mathcal{O}\left(g^{4}\right)$.

The mixing of $O_{g}$ into $\tilde{O}_{Q D}$ has not been considered so far because it arises from one-loop diagrams with external quantum gluon legs. We need to consider such diagrams as they are divergent subdiagrams in the further two-loop calculation.

One may ask why only the very operators $\tilde{O}_{D D}$ and $\tilde{O}_{Q D}$ arise as nonphysical counterterms. We know [9] that all such counterterms either have to vanish by the EOM or be BRS-exact. Both $\tilde{O}_{D D}$ and $\tilde{O}_{Q D}$ vanish by the EOM for the quarks. In our case, there is not "enough dimension" for the EOM for the gauge bosons (such EOM has dimension 3 and the two quark fields have also dimension 3). So any EOM-vanishing operator must be proportional to the EOM for a quark. Two quark fields and a covariant derivative together have dimension 4. The remaining one unit of dimension can be provided either by another covariant derivative (then the only possibility is $\tilde{O}_{D D}$ ) or by the quantum gluon field (as in $\tilde{O}_{Q D}$ ). The background gluon field cannot show up because our operators must be invariant under the gauge transformation given in eqs. (2.14) and (2.15) of ref. 81: 8

$$
\begin{aligned}
\delta A_{\mu}^{a} & =-f^{a b c} \omega^{b} A_{\mu}^{c}-\frac{1}{g} \partial_{\mu} \omega^{a} \\
\delta Q_{\mu}^{a} & =-f^{a b c} \omega^{b} Q_{\mu}^{c}
\end{aligned}
$$

where $\omega^{a}$ denotes the gauge transformation parameter. The two above transformations combine to the usual gauge transformation for the full gluon field $A_{\mu}^{a}+Q_{\mu}^{a}$.

The final observation is that our initial operators $O_{\gamma}$ and $O_{g}$ are odd under charge conjugation combined with exchange of $q$ and $q^{\prime}$. This is why the two terms in $\tilde{O}_{Q D}$ come in a combination odd under this symmetry.

BRS-exact operators cannot arise as counterterms to $O_{\gamma}$ and $O_{g}$. The arguments for this are the following: The BRS variation raises both dimension and ghost number by one unit. So any BRS-exact operator that could mix with $O_{\gamma}$ or $O_{g}$ should be a BRS variation of a dimension-four operator with ghost number -1 . The only possibility for the latter operator is $\bar{\eta}^{a} \bar{q} T^{a} q^{\prime}$. The BRS variation of such an operator contains a term with two fermions and two ghosts. On the other hand, such a term cannot arise as a counterterm for the magnetic moment

\footnotetext{
${ }^{2}$ In our conventions, the sign of $g$ is opposite to that in ref. [8].
} 
operator because the corresponding diagram in fig. 2 is convergent. [1

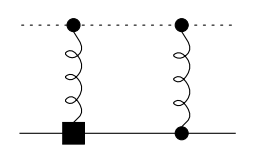

Figure 2: Convergent diagram for the $q^{\prime} \rightarrow q \bar{\eta} \eta$ transition.

As follows from the above considerations, one can show that $O_{\gamma}, O_{g}, \tilde{O}_{D D}$ and $\tilde{O}_{Q D}$ are the only possible counterterms for $O_{\gamma}$ and $O_{g}$ without calculating a single Feynman diagram. Then the matrix in eq. (7) can be found just from the diagrams depicted in fig. 11 with only quantum gluon on external lines. The calculation is then identical to the one in the usual Feynman-'t Hooft gauge (without background field).

Since our calculation was fully computerized, it did not require much effort to check that indeed all the divergences in the diagrams with single $O_{\gamma}$ and $O_{g}$ insertions (also diagrams with two external gauge bosons) can be renormalized by the same counterterms proportional to $O_{\gamma}$, $O_{g}, \tilde{O}_{D D}$ and $\tilde{O}_{Q D}$. This was one of the cross-checks in our calculation.

The last remark in order is that the operator $\tilde{O}_{Q D}$ would arise also in the non-backgroundfield calculation. Then $Q$ in $\tilde{O}_{Q D}$ would stand for the full gluon field. Consequently, this operator would not be gauge-invariant (not even BRS-invariant). However, this is not a problem because EOM-vanishing operators are nonphysical anyway, i.e. their physical matrix elements vanish. They do not mix into physical operators, either [9]. This is why the unknown entries in the lower right corner of the matrix in eq. (7) are completely irrelevant.

One may then wonder why we have decided to recover of the mixing of $O_{g}$ into $\tilde{O}_{D D}$ and $\tilde{O}_{Q D}$ at all. The reason for this is the method we have applied for calculating the two-loop diagrams in the next section.

\section{The two-loop calculation}

At the two-loop level we need to calculate the $q^{\prime} \rightarrow q \gamma$ and $q^{\prime} \rightarrow q A$ Green's functions with single $O_{\gamma}$ or $O_{g}$ insertions. Their divergent parts give the two-loop mixing among these operators. As a by-product, we obtain the two-loop mixing of $O_{g}$ into $\tilde{O}_{D D}$. The latter mixing must come out

\footnotetext{
${ }^{3}$ At first glance, this diagram has zero degree of divergence (remember that the operator vertex has dimension 1 ). But one power of momentum in the power counting is only the momentum of the outgoing external ghost - just because of the structure of the gluon-ghost vertex. So the effective degree of divergence is -1 . This argument applies to all orders.
} 
the same both from photonic and gluonic Green's functions. This is a nontrivial cross-check of the calculation.

The main problem in the two-loop calculation is the large number of diagrams to be considered. For instance, there are 167 two-loop diagrams for the self-mixing of $O_{g}$. Therefore, a simple systematic algorithm is necessary to make the calculation fully computerized. With such an algorithm it is not really important whether one has to calculate 100 or 1000 two-loop diagrams - it is only a matter of computer time which in our particular case was around two days (on a SUN ELC workstation).

The input to our programs were the Feynman rules following from the Lagrangian in eq. (4). The program FeynArts [11] was used for constructing the relevant topologies (see fig. 3), drawing the diagrams and creating the analytic expressions for the corresponding amplitudes.

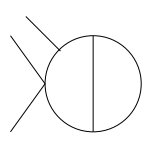

(11)

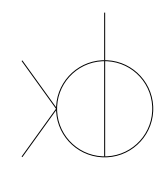

(2)

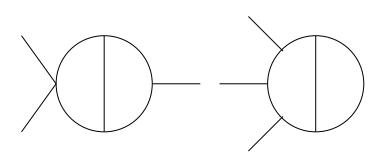

(7)

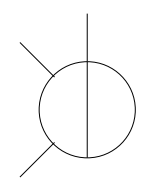

(18)

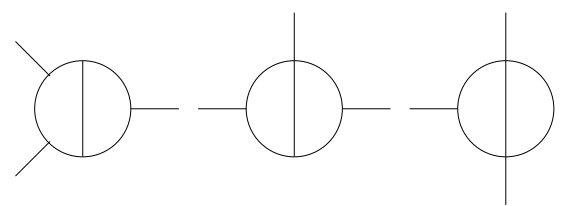

$(51)$

(12)

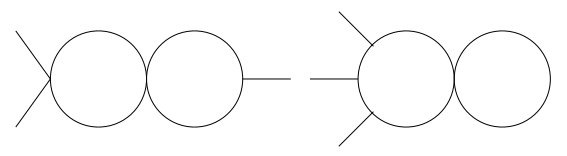

(1)
(10)

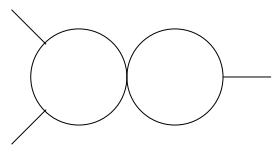

(7)

Figure 3: Topologies of the Feynman diagrams for the two-loop self-mixing of $O_{g}$. The numbers in brackets are the numbers of diagrams corresponding to each topology.

Next, a single algorithm (around ten pages of Mathematica 12 codet) was applied to each of the diagrams. The potentially UV-divergent expressions were expanded to first order in external momenta and then the tensor integrals were reduced to scalar ones. So all the integrals we needed to calculate were scalar two-loop integrals independent of external momenta.

There were no physical masses in our calculation. But before the expansion in the external momenta was made, an auxiliary mass was introduced in all the propagator denominators. Otherwise expanding in external momenta would create spurious infrared divergences that would be indistinguishable from the UV-divergences within the framework of dimensional regularization.

\footnotetext{
${ }^{4}$ We had two programs created independently by each of us.
} 
These infrared divergences would not cancel in the sum of all the diagrams.

The auxiliary mass was the same for all the propagator denominators. So all the two-loop scalar integrals we needed to calculate were of the form

$$
I_{a b c}=\int \frac{d^{D} q}{(2 \pi)^{D}} \frac{d^{D} r}{(2 \pi)^{D}} \frac{1}{\left[q^{2}-m^{2}\right]^{a}\left[r^{2}-m^{2}\right]^{b}\left[(q+r)^{2}-m^{2}\right]^{c}}
$$

where $a, b$ and $c$ are integers and $\mathrm{D}$ is the dimensionality of spacetime. A change of variables $(q \rightarrow q+r, r \rightarrow-r)$ shows that $I_{a b c}$ is symmetric under permutations of $a, b$ and $c$. If any of these integers is nonpositive, the integral is easily reduced to a product of one-loop integrals. For $a, b$ and $c$ all positive one has

$$
\begin{aligned}
& I_{111}=-\frac{3 m^{2}}{(4 \pi)^{4}}\left[\frac{2}{\epsilon^{2}}+\frac{1}{\epsilon}\left(3-2 \gamma_{E}-2 \ln \frac{m^{2}}{4 \pi}\right)\right]+\text { convergent terms } \\
& I_{211}=-\frac{1}{(4 \pi)^{4}}\left[\frac{2}{\epsilon^{2}}+\frac{1}{\epsilon}\left(1-2 \gamma_{E}-2 \ln \frac{m^{2}}{4 \pi}\right)\right]+\text { convergent terms } \\
& I_{n 11}=\frac{(-1)^{n+1}}{(4 \pi)^{4} \epsilon} \frac{2}{(n-1)(n-2) m^{2(n-2)}}+\text { convergent terms, for } n \geq 3 .
\end{aligned}
$$

where $\epsilon=4-D$. When $a, b$ and $c$ are positive and two of them are greater than 1 , then $I_{a b c}$ is convergent. This fact and the above three equations are derived in the appendix.

Eqs. (11)-(13) together with similar expressions for one-loop scalar integrals reduce the calculation of the divergent part of any integral $I_{a b c}$ in eq. (10) to simple algebra. The further steps of the calculation are of course purely algebraic, too: The results of the scalar integrals were combined back into tensor integrals and then contracted with the Dirac matrices, partly using Tracer [13]. Performing the Dirac algebra was straightforward in our case, as we had only a single open fermion line and no $\gamma_{5}$ in our calculation. So no "evanescent operators" [14] had to be introduced.

Before presenting our final results, we should give arguments why introducing an auxiliary mass in all the propagator denominators and then expanding in external momenta leads us to the same results for the divergent part of the sum of all the diagrams as we would get without introducing this auxiliary mass and without expanding.

The key point is the well-known fact that once all the subdivergences are removed in a mass independent renormalization scheme, the pole part part of a dimensionally regularized

\footnotetext{
${ }^{5}$ The simplest way to convince oneself about this is to consider an example with only one relevant diagram, e.g. one-loop wave-function renormalization in the scalar massless $\lambda \phi^{3}$ theory.

${ }^{6}$ i.e. the sum of the two-loop diagrams and the one-loop counterterm diagrams which remove subdivergences
} 
Feynman diagram is polynomial in masses and external momenta. This fact can be shown on the basis of given Feynman rules alone, and no field-theoretical arguments have to be used. The proof [9] is based on Weinberg's theorem [15] and on the property, that differentiation with respect to external momenta decreases the degree of divergence of a Feynman diagram with no subdivergences. Adding auxiliary masses in propagator denominators does not affect this proof at all. So, after introducing these masses, the pole part of a Feynman diagram with subtracted subdivergences still will be a polynomial in external momenta and all the masses (including the auxiliary ones). Therefore, setting the auxiliary masses to zero in the end, we recover the result for the pole part of the diagram with no auxiliary masses. However, the latter is true only for the pole part and only after subtraction of subdivergences.

The one-loop diagrams with counterterm vertices that cancel the subdivergences in two-loop diagrams have to be calculated with exactly the same Feynman rules as the two-loop diagrams. The same refers to the calculation of the one-loop renormalization constants themselves. So we also need to use massive propagators e.g. in all the diagrams for the one-loop renormalization of the two-point Green's function for the gluon. This necessarily leads to introduction of a "gluon mass" that gets renormalized at the one-loop level. It is known 16] that a theory with a bare mass for a vector boson has no physical sense.] But so long as the would-be gauge-fixing term is present in the Lagrangian, it is renormalizable by power counting [17], i.e. the Green's functions (even if physically meaningless) can be rendered finite with help of local counterterms. Moreover, in a mass-independent renormalization scheme (such as the $\overline{M S}$ scheme we use), these counterterms will be exactly the same as in the massless case (except for the renormalization of the vector boson mass itself). So the Feynman rules for the physically meaningless theory with a massive gluon can be used for recovering renormalization constants in true QCD.

Similar arguments can be used to justify that we could introduce an auxiliary mass in all fermion propagator denominators without introducing it also in the numerators. Needless to say, this also affects the calculation of the "gluon mass" counterterm.

Introducing auxiliary masses does not affect the results for the one-loop mixing summarized in eq. (7). Neither it affects the usual QCD renormalization constants (except for renormalization of mass terms). However, it affects the role the EOM-vanishing operators $\tilde{O}_{D D}$ and $\tilde{O}_{Q D}$

\footnotetext{
${ }^{7}$ Unless this boson couples only to conserved currents.
} 
play in our calculation. With the modified Feynman rules, the usual arguments which show that on-shell matrix elements of these operators vanish no longer hold. These operators remain nonphysical in the end, when we set the auxiliary masses to zero. But they are important at the intermediate stages of our calculation, i.e. the one-loop counterterm diagrams with counterterms proportional to $\tilde{O}_{D D}$ and $\tilde{O}_{Q D}$ do affect the mixing among the physical operators $O_{\gamma}$ and $O_{g}$.

A remark concerning expansion in external momenta is now in order. As the two-loop diagrams for $q^{\prime} \rightarrow q \gamma$ and $q^{\prime} \rightarrow q A$ transitions have degree of divergence equal to +1 , the pole parts of our diagrams are linear in external momenta. This is why we expand in external momenta only to the first order before performing the loop integrations. The expansion in external momenta can be viewed as an exact splitting of propagators into parts that are polynomial in external momenta and parts that contribute to integrands with lower degree of divergence, as in the following example:

$$
\frac{1}{(q+p)^{2}-m^{2}}=\frac{1}{q^{2}-m^{2}}+\frac{-2 q p-p^{2}}{q^{2}-m^{2}} \frac{1}{(q+p)^{2}-m^{2}}
$$

where $p$ denotes the external momentum and $q$ is the loop momentum. Performing such an operation appropriately many times (in a diagram with subtracted subdivergences) one can split the integral into a convergent part and a part that is a polynomial in external momenta. This shows that for the pole part of a diagram with subtracted subdivergences, the expansion in external momenta is allowed before the loop integration is made.

Our final results were subject to several cross-checks. First, the double pole part of the sum of all the two-loop diagrams had to be twice smaller and have the opposite sign than the double-pole part of the sum of all the one-loop diagrams with counterterm vertices. Only then the sum of all the diagrams together can be local (i.e. polynomial in external momenta). Second, the pole parts needed to have the structure of the gauge-invariant operators $O_{\gamma}, O_{g}$ and $\tilde{O}_{D D}$ (the operator $\tilde{O}_{Q D}$ could not appear as we have not calculated two-loop diagrams with an external quantum gluon). This led to relations between coefficients at different Lorentz structures (usually two relations for four independent structures). Next, the mixing of $O_{g}$ into $\tilde{O}_{D D}$ had to come out the same from both $q^{\prime} \rightarrow q \gamma$ and $q^{\prime} \rightarrow q A$ diagrams. This is also a manifestation of gauge invariance. It is interesting to note that the latter requirement was fulfilled only after proper inclusion of the "gluon mass" one-loop counterterms. Finally, the 
two-loop self mixing of $O_{\gamma}$ which is given by a relatively small number of diagrams has been calculated also "by hand" without introducing auxiliary masses and expanding in the external momenta.

The final result of our two-loop calculation is the explicit expression for the $\mathcal{O}\left(g^{4}\right)$ part of the matrix $\hat{\gamma}$ in eq. ([) . The anomalous dimension matrix now reads

$$
\hat{\gamma}=\frac{g^{2}}{16 \pi^{2}}\left(\begin{array}{cc|cc}
2 C_{f} & 0 & 0 & 0 \\
8 C_{f} Q_{q} & 10 C_{f}-4 N & -12 C_{f} & \frac{3}{2} N \\
\hline 0 & 0 & ? & ? \\
0 & 0 & ? & ?
\end{array}\right)+\frac{g^{4}}{\left(16 \pi^{2}\right)^{2}}\left(\begin{array}{cc|cc}
a & 0 & 0 & 0 \\
b & c & d & ? \\
\hline 0 & 0 & ? & ? \\
0 & 0 & ? & ?
\end{array}\right)+\mathcal{O}\left(g^{6}\right)
$$

where

$$
\begin{aligned}
a & =C_{f}\left(\frac{257}{9} N-19 C_{f}-\frac{26}{9} f\right) \\
b & =C_{f}\left(\frac{404}{9} N-32 C_{f}-\frac{56}{9} f\right) Q_{q}, \\
c & =-\frac{299}{9}-\frac{51}{4 N^{2}}+\frac{247}{36} N^{2}+\frac{41}{9} \frac{f}{N}+\frac{2}{9} f N
\end{aligned}
$$

and

$$
d=C_{f}\left(-\frac{643}{6} N+60 C_{f}+\frac{44}{3} f\right)
$$

Let us now briefly come to the case of two identical quark flavors, $q \equiv q^{\prime}$. Then we have to

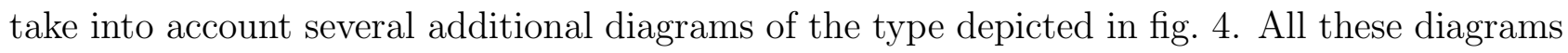
contain a trace over an odd number of Dirac matrices (remember we are working with massless quarks) and so give no contribution. Thus, the results (15)-(19) can be directly used for flavor-conserving magnetic moment operators as well. $0^{0}$

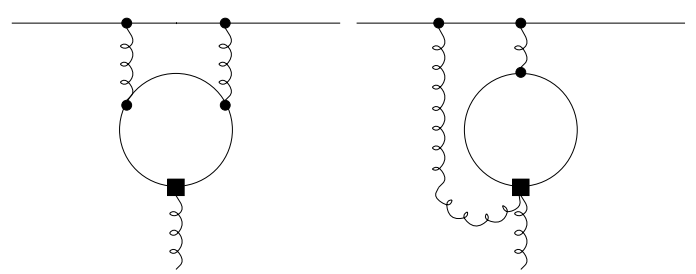

Figure 4: Examples of additional diagrams for flavor-conserving operators.

\footnotetext{
${ }^{8}$ We would like to thank Matthias Jamin for bringing this fact to our attention.
} 


\section{The $b \rightarrow s \gamma$ case}

Having in mind the potential application of our results to the calculation of the next-to-leading logarithmic corrections to the $B \rightarrow X_{s} \gamma$ decay rate, we now rewrite the $2 \times 2$ anomalous dimension matrix of the magnetic moment operators once again in the conventional notation used in the analyses of this process. If we normalize the two magnetic moment operators as in ref. [7]

$$
\begin{aligned}
Q_{7} & =\frac{e}{8 \pi^{2}} m_{b} \bar{s}_{\alpha} \sigma^{\mu \nu}\left(1+\gamma_{5}\right) b^{\alpha} F_{\mu \nu} \\
Q_{8} & =\frac{g}{8 \pi^{2}} m_{b} \bar{s}_{\alpha} \sigma^{\mu \nu}\left(1+\gamma_{5}\right)\left(T^{a}\right)_{\beta}^{\alpha} b^{\beta} G_{\mu \nu}^{a}
\end{aligned}
$$

then their $2 \times 2$ anomalous dimension matrix can be obtained from the corresponding entries in eqs. (15)-(16) just by subtracting $\gamma_{m}$ from the diagonal terms. At the two-loop level, the anomalous dimension of the mass reads 18 with our sign convention

$$
\gamma_{m}=-\frac{g^{2}}{16 \pi^{2}} 6 C_{f}+\frac{g^{4}}{\left(16 \pi^{2}\right)^{2}} C_{f}\left(-\frac{97}{3} N-3 C_{f}+\frac{10}{3} f\right)+\mathcal{O}\left(g^{6}\right) .
$$

Consequently, the anomalous dimension matrix for $Q_{7}$ and $Q_{8}$ is

$$
\hat{\gamma}(g)=\hat{\gamma}^{(0)} \frac{g^{2}}{16 \pi^{2}}+\hat{\gamma}^{(1)} \frac{g^{4}}{\left(16 \pi^{2}\right)^{2}}+\mathcal{O}\left(g^{6}\right)
$$

where

$$
\hat{\gamma}^{(0)}=\left(\begin{array}{lc}
8 C_{f} & 0 \\
8 C_{f} Q_{s} & 16 C_{f}-4 N
\end{array}\right)=\left(\begin{array}{rr}
\frac{32}{3} & 0 \\
-\frac{32}{9} & \frac{28}{3}
\end{array}\right)
$$

and, in the $\overline{M S}$ scheme,

$$
\begin{gathered}
\hat{\gamma}^{(1)}=\left(\begin{array}{cc}
C_{f}\left(\frac{548}{9} N-16 C_{f}-\frac{56}{9} f\right) & 0 \\
Q_{s} C_{f}\left(\frac{404}{9} N-32 C_{f}-\frac{56}{9} f\right) & -\frac{458}{9}-\frac{12}{N^{2}}+\frac{214}{9} N^{2}+\frac{56}{9} \frac{f}{N}-\frac{13}{9} f N
\end{array}\right)= \\
=\left(\begin{array}{cc}
\frac{4688}{27} & 0 \\
-\frac{2192}{81} & \frac{4063}{27}
\end{array}\right) .
\end{gathered}
$$

Unfortunately, knowing the above anomalous dimension matrix is not enough to write the full next-to-leading RGE for the coefficients of $Q_{7}$ and $Q_{8}$. As explained in detail in ref. [7], the three-loop mixing of the four-quark operators into the magnetic moment operators gives contributions of the same order as the two-loop self-mixing of the latter operators. Therefore, our calculation is an important step towards the next-to-leading calculation of the $B \rightarrow X_{s} \gamma$ 
rate, but by no means completes this calculation. We hope that the methods described in the present paper can provide helpful tools in calculating the desired three-loop mixing.

The numerical value of the next-to-leading corrections we have calculated can be easily determined from the matrix in eq. (25) and the analysis presented in ref. [7]. The equations (4)-(9) of that paper summarize the leading-order expressions for the $b \rightarrow s \gamma$ decay rate which is proportional to the square of the quantity denoted there by $C_{7}^{(0) e f f}$. Inserting the matrices from eqs. (24) and (25) above into the equations (21)-(28) of ref. [7], we find the next-to-leading contribution that adds to $C_{7}^{(0) e f f}$ in the $\overline{M S}$-scheme:

$$
\begin{aligned}
& \Delta C_{7}^{e f f}(\mu)=\frac{\alpha_{s}\left(M_{W}\right)}{4 \pi}\left[\frac{37208}{4761}\left(\eta^{\frac{16}{23}}-\eta^{-\frac{7}{23}}\right) C_{7}^{(0)}\left(M_{W}\right)+\right. \\
& \left.\quad+\left(\frac{297664}{14283} \eta^{-\frac{7}{23}}-\frac{7164416}{357075} \eta^{-\frac{9}{23}}+\frac{256868}{14283} \eta^{\frac{14}{23}}-\frac{6698884}{357075} \eta^{\frac{16}{23}}\right) C_{8}^{(0)}\left(M_{W}\right)\right],
\end{aligned}
$$

with $\eta, C_{7}^{(0)}\left(M_{W}\right)$ and $C_{8}^{(0)}\left(M_{W}\right)$ defined as in eqs. (5), (8) and (9) of ref. [7]. For $\alpha_{s}\left(M_{Z}\right)=0.12$, $m_{t}=174 \mathrm{GeV}$ and $\mu=5 \mathrm{GeV}$, the above contribution causes a decrease of the predicted branching ratio by around 5\%. However, no phenomenological conclusions can be drawn before all the other next-to-leading corrections are calculated. Only then the total correction is renormalization-scheme independent.

The leading order expression for the coefficient of the gluonic operator is given in the last equation of ref. [7]. The next-to-leading contribution from the two-loop self-mixing of $Q_{8}$ reads (in the $\overline{M S}$-scheme)

$$
\Delta C_{8}^{e f f}(\mu)=\frac{\alpha_{s}\left(M_{W}\right)}{4 \pi} \frac{64217}{9522}\left(\eta^{\frac{14}{23}}-\eta^{-\frac{9}{23}}\right) C_{8}^{(0)}\left(M_{W}\right)
$$

which in the Standard Model case gives numerically around 2.4\% decrease of the coefficient, i.e. a similar change as in the photonic case. Even when the full next-to-leading calculation is completed, its effect on the coefficient of the gluonic operator is not expected to have any measurable physical consequences. So we should treat eq. (27) only as a by-product of the calculation performed for the photonic operator $Q_{7}$.

In the end, we would like to point out once again that our two-loop anomalous dimension matrix is insensitive to the scheme used for $\gamma_{5}$ in dimensional regularization. Two-loop mixing usually depends on the scheme used for $\gamma_{5}$, and the scheme dependence of the two-loop anomalous dimension matrix cancels in physical quantities with the scheme dependence of the 
one-loop matrix elements [3-6]. In the case of $Q_{7}-Q_{8}$ mixing, we have completely eliminated $\gamma_{5}$ from the calculation by using chirality conservation in QCD and QED interactions. This was equivalent to a trivial application of the recent Pisa scheme for $\gamma_{5}$ [5]. However, exactly the same anomalous dimension matrix would be obtained in any of the other commonly used schemes HV, NDR and DRED (see Buras and Weisz in ref. [3] for a summary of these schemes). So the situation here is much simpler than in the case of four-quark operators.

To conclude: We have calculated the two-loop QCD mixing of the magnetic moment operators which are the only EOM-nonvanishing dimension-five flavor-changing operators that arise in the Standard Model after integrating out the $W, Z$ and Higgs bosons and the top-quark. Our calculation is an important contribution to the phenomenologically desired next-to-leading QCD corrections to the $b \rightarrow s \gamma$ decay. This was the last two-loop QCD mixing to be calculated for the effective hamiltonian describing flavor changing processes at low energies in the Standard Model and many of its extensions.

\section{Acknowledgements}

We would like to thank Andrzej Buras, Matthias Jamin, Manfred Lindner, Uli Nierste, Hubert Simma and Peter Weisz for helpful discussions. This research has been supported by the German Bundesministerium für Forschung und Technologie under contract 06 TM 732, by the CEC Science project SC1-CT91-0729 and by the Polish Committee for Scientific Research.

\section{Appendix}

In order to derive the equations (11)-(13) one starts from the integral

$$
J_{211}\left(M, m_{1}, m_{2}\right)=\int \frac{d^{D} q}{(2 \pi)^{D}} \frac{d^{D} r}{(2 \pi)^{D}} \frac{1}{\left[q^{2}-M^{2}\right]^{2}\left[r^{2}-m_{1}^{2}\right]\left[(q+r)^{2}-m_{2}^{2}\right]} .
$$

Differentiating the integrand with respect to $m_{1}$ or $m_{2}$ we find an integral that is convergent by Weinberg's theorem [15] (i.e. all the subdiagrams of the corresponding scalar diagram have negative degree of divergence). Consequently, the divergent part of $J_{211}\left(M, m_{1}, m_{2}\right)$ is independent of $m_{1}$ and $m_{2}$. We can therefore replace $J_{211}\left(M, m_{1}, m_{2}\right)$ by $J_{211}(M, 0,0)$ and easily calculate the latter with help of the Feynman parametrization

$$
J_{211}\left(M, m_{1}, m_{2}\right)=J_{211}(M, 0,0)+\text { convergent terms }=
$$




$$
=-\frac{1}{(4 \pi)^{4}}\left[\frac{2}{\epsilon^{2}}+\frac{1}{\epsilon}\left(1-2 \gamma_{E}-2 \ln \frac{M^{2}}{4 \pi}\right)\right]+\text { convergent terms }
$$

By integrating and/or differentiating the above result with respect to the masses appropriately many times and then setting all the masses equal, we obtain the equations (11)-(13). This is also how one learns that $I_{a b c}$ is convergent when two of its indices are greater than 1 and all of them are positive.

The short derivation presented in this appendix is motivated by the appendix of ref. [19] where also the finite parts of the integrals are given. However, we disagree with the global sign of the explicit expression for $\left(M, M\left|M_{1}\right| M_{2}\right)$ given there.

\section{References}

[1] see e.g. A. J. Buras and M. K. Harlander, in "Heavy Flavors", eds. A. J. Buras and M. Lindner, World Scientific Publ. Co., Singapore 1992, p. 58.

[2] B. Grinstein, R. Springer and M.B. Wise, Nucl. Phys. B339 (1990) 269

[3] G. Altarelli, G. Curci, G. Martinelli and S. Petrarca, Nucl. Phys. B187 (1981) 461;

A. J. Buras and P. H. Weisz, Nucl. Phys. B333 (1990) 66;

A. J. Buras, M. Jamin, M. E. Lautenbacher and P. H. Weisz, Nucl. Phys. B400 (1993) 37;

A. J. Buras, M. Jamin and M. E. Lautenbacher, Nucl. Phys. B400 (1993) 75;

M. Ciuchini E. Franco, G. Martinelli and L. Reina, Nucl. Phys. B415 (1994) 403

[4] M. Misiak, Nucl. Phys. B393 (1993) 23;

A. J. Buras, M. E. Lautenbacher, M. Misiak and M. Münz, Nucl. Phys. B423 (1994) 349

[5] G. Cella, G. Curci, G. Ricciardi and A. Viceré, Phys. Lett. B248 (1990) 181;

Phys. Lett. B325 (1994) 227; preprint IFUP-TH 9/94, hep-ph/9406203

[6] R. Grigjanis, P.J. O’Donnell and M. Sutherland and H. Navelet, Phys. Lett. B213 (1988) 355; Phys. Lett. B286 (1992) 413E;

M. Misiak, Phys. Lett. B269 (1991) 161; Nucl. Phys. B393 (1993) 23; Phys. Lett. B321 (1994) 113;

K. Adel and Y.P. Yao, Mod. Phys. Lett. A8 (1993) 1679; Phys. Rev. D49 (1994) 4945;

M. Ciuchini, E. Franco, G. Martinelli, L. Reina and L. Silvestrini, Phys. Lett. B316 (1993) 127;

M. Ciuchini, E. Franco, L. Reina and L. Silvestrini, Nucl. Phys. B421 (1994) 41

[7] A. J. Buras, M. Misiak, M. Münz and S. Pokorski, Nucl. Phys. B424 (1994) 374

[8] L. F. Abbott, Nucl. Phys. B185 (1981) 189

[9] J. C. Collins, "Renormalization", Cambridge Univ. Press, Cambridge 1984;

see also J. C. Collins and R. J. Scalise, preprint PSU/TH/141, hep-ph/9403231;

B. W. Harris and J. Smith, preprint ITP-SB-94-39, hep-ph/9409405 
[10] M. A. Shifman, A. I. Vainstein and V. I. Zakharov, Phys. Rev. D18 (1978) 2583

[11] J. Küblbeck, M. Böhm and A. Denner, Comput. Phys. Commun. 60 (1990) 165

[12] S. Wolfram, Mathematica, Addison-Wesley Publ. Comp., N.Y. 1988.

[13] M. Jamin and M. E. Lautenbacher, Comput. Phys. Commun. 74 (1993) 265

[14] M. J. Dugan and B. Grinstein, Phys. Lett. B256 (1991) 239

[15] S. Weinberg, Phys. Rev. 118 (1960) 838

[16] J. Zinn-Justin, "Quantum field theory and critical phenomena", Clarendon press, Oxford 1989, chapter 17

[17] C. Itzykson and J. B. Zuber, "Quantum field theory", McGraw-Hill Inc. 1980, sect. 3-2-3

[18] P. Pascual and R. Tarrach, "QCD: Renormalization for the practitioner", Springer-Verlag, Berlin-Heidelberg 1984

[19] J. van der Bij and M. Veltman, Nucl. Phys. B231 (1984) 205 